\title{
Second targeted combination regime approved for metastatic melanoma
}

$\mathrm{R}$ esearchers are increasingly looking to rational combinations of drugs to meet the challenge of resistance to molecularly targeted anticancer therapies. The treatment of metastatic melanoma was significantly advanced with the development of BRAF inhibitors, but is still limited by the short-lived nature of the responses to these drugs and the almost universal occurrence of tumor regrowth. More durable responses could be produced by targeting multiple points in the mitogen-activated protein kinase (MAPK) signaling pathway of which BRAF is a central component. In the fall of 2015, the MEK inhibitor cobimetinib and BRAF inhibitor vemurafenib became the second such combination to receive regulatory approval in the United States following positive results from the international, phase 3 , randomized coBRIM trial, which demonstrated the superiority of the combination over singleagent BRAF inhibitor therapy. It also marked the first US approval for cobimetinib. ${ }^{1}$

A total of 495 patients aged 18 years or older, with histologically confirmed, resectable, locally advanced stage IIIC or IV melanoma harboring BRAF V600 mutations (as detected by the Cobas 4800 BRAF v600 Mutation Test) were enrolled at 135 sites in the United States, Canada, Australia, New Zealand, Europe, Russia, Turkey, and Israel from January 2013-January 2014. In addition, patients had measurable disease according to Response Evaluation Criteria in Solid Tumors (RECIST, version 1.1), as assessed by computed tomography; an Eastern Cooperative Oncology Group (ECOG) Scale performance status score of 0 (fully active and able to carry on all performance without restriction) or 1 (restricted in physically strenuous activity but ambulatory and able to carry out work of a light or sedentary nature); adequate hematologic, hepatic, renal, and cardiac function; and, in those with brain metastases, at least a 3-week history of stable disease.

Patients were randomized 1:1 to receive oral vemurafenib $960 \mathrm{mg}$ twice daily, with either placebo or oral cobimetinib $60 \mathrm{mg}$ once daily (on a 21-day on, 7-day off cycle) until withdrawal of consent, unacceptable toxicity, or disease progression. Randomization was stratified according to American Joint Committee on Cancer stage and geographic region. Tumor assessments were performed at baseline and every 8 weeks. Baseline patient characteristics were well balanced for age, ECOG performance status,

\section{What's new, what's important}

In 2015, the FDA approved cobimetinib, a novel MEK inhibitor, in combination with the BRAF inhibitor, vemurafenib for the treatment of advanced, unresectable or metastatic melanoma that has BRAF V600E or V600K mutation.

The safety and efficacy of cobimetinib taken in combination with vemurafenib were demonstrated in a randomized clinical study of 495 patients with previously untreated, BRAF V600 mutation-positive melanoma. The combination therapy had a $70 \%$ response rate (partial or complete response), compared with $50 \%$ for single agent vemurafenib. The combination therapy also showed improvement in progression-free survival and overall survival.

The recommended dose of cobimetinib is $60 \mathrm{mg}$ orally once daily for the first 21 days of a 28-day cycle and the dose of vemurafenib is $960 \mathrm{mg}$ twice daily until disease progression or unacceptable toxicity.

The most common side effects of the combination therapy includes diarrhea, sensitivity to UV light, photosensitivity reaction, nausea, fever and vomiting. Cobimetinib can also cause cardiomyopathy or rhabdomyolysis, primary cutaneous malignancies, retinal detachment, severe skin rash, hepatotoxicity, photosensitivity. Patients who are taking cobimetinib should avoid sun exposure, wear protective clothing, and a broad spectrum UVA/AVB sunscreen to protect against sunburn.

This combination treatment is giving more treatment options for patients with metastatic melanoma.

- Jame Abraham, MD, FACP (abrahaj5@ccf.org)

geographic region, and disease stage. More than half of the patients had stage IV M1c melanoma.

The primary endpoint was investigator-assessed progression-free survival (PFS), according to RECIST (version 1.1). According to updated data presented at the 2015 annual meeting of the American Society of Clinical Oncology (ASCO), the combination of cobimetinib and vemurafenib significantly improved PFS compared with vemurafenib and placebo (12.3 months vs 7.2 months, respectively; hazard ratio $[\mathrm{HR}], 0.58 ; P<.001)$, and the benefit was observed across all prespecified patient subgroups.

Secondary endpoints included overall survival (OS), confirmed objective response rate (ORR), duration of response (DoR), PFS as assessed by independent review

Report prepared by Jane De Lartigue, PhD. JCSO 2016;14:51-53. @2016 Frontline Medical Communications. doi:10.12788/icso.0234. 


\section{Mechanism of action - cobimetinib and vemurafenib}

\section{A double blow to MAPK signaling}

The treatment of metastatic melanoma has been revolutionized by the development of drugs targeting the BRAF kinase, a key component of the MAPK pathway. This signaling cascade plays a central role in promoting cell proliferation and survival through the activation of a series of kinases, culminating in the translocation of ERK $1 / 2$ to the nucleus, which stimulates the transcription of a plethora of gene targets, including cyclin D1 (CCNDI).

The MAPK pathway is corrupted as a means of cancer cell growth in more than $80 \%$ of primary cutaneous melanomas, primarily as a result of mutations in the BRAF gene. In particular, V600E/K mutations in BRAF have a well-delineated role as oncogenes in melanoma, serving as the stimulus for significant investment in the development of small molecule inhibitors of this kinase.

Vemurafenib is a BRAF inhibitor that received regulatory approval from the FDA in 2011 for the treatment of BRAF-mutant metastatic melanoma, following the demonstration of improved progression-free and overall survival compared with chemotherapy. Although it represented a significant advancement for the treatment of this tumor type, $5 \%-20 \%$ of patients don't respond and those who do invariably experience tumor regrowth in less than a year.

A wide variety of molecular mechanisms rendering tumors insensitive to BRAF inhibitors have been revealed, many of which involve continued dependence on the MAPK pathway, implying that drugs that employ a secondary hit on this signaling network may be a particularly effective therapeutic strategy.

Cobimetinib is a highly specific, reversible inhibitor of the MEK $1 / 2$ kinases that function downstream of BRAF. Its approval in combination with vemurafenib marks a first for this

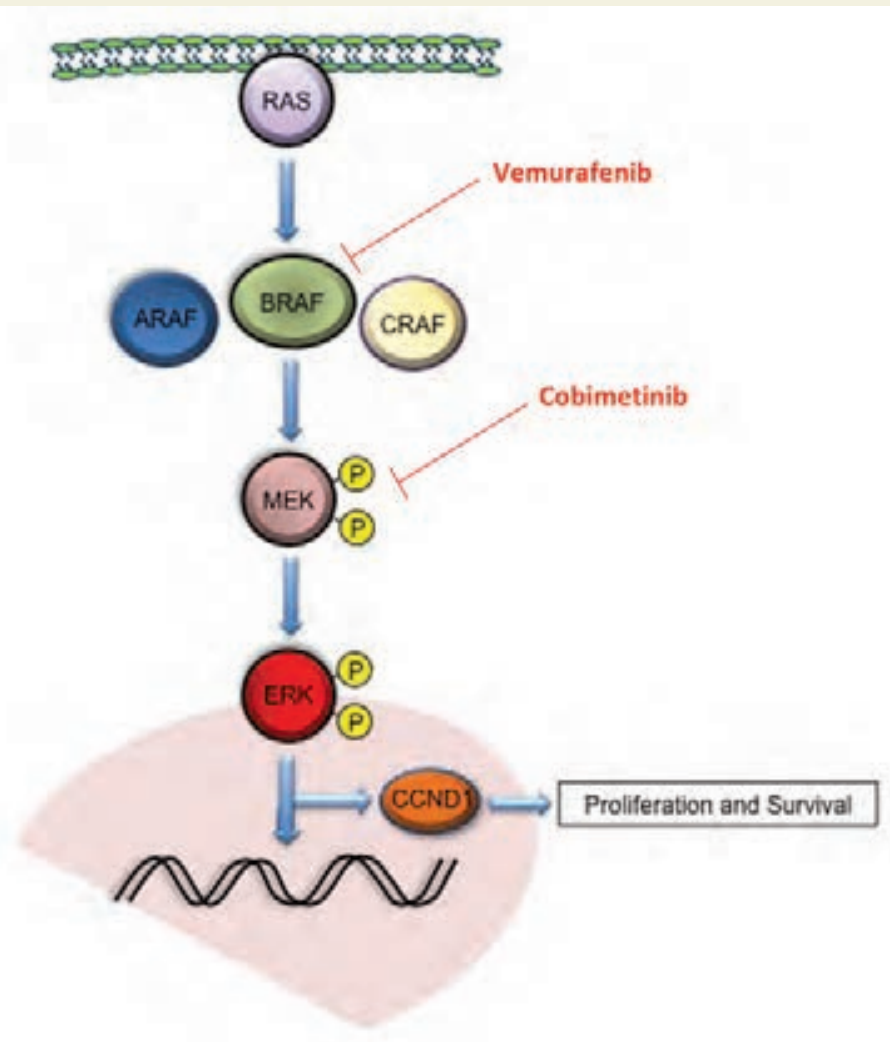

Combined BRAF (vemurafenib) and MEK (cobimetinib) inhibition hits the MAPK pathway, which is constitutively activated in the majority of melanomas, at 2 different points to overcome resistance and produce more durable responses.

Reproduced with permission; Gowrishankar et al. ${ }^{5}$

drug and a second approval for a combination of BRAF and MEK inhibitors, following on from the successful development of dabrafenib and trametinib in 2014. Cotargeting 2 components of the MAPK pathway may help to manage and prevent BRAF inhibitor resistance. facility, and safety. At the time of final analysis in the pivotal trial, median OS had not been reached in either group. The 9 -month OS rate was $81 \%$ and $73 \%$ for the combination and control groups, respectively, but did not cross the prespecified HR boundary for significance. The companies jointly developing cobimetinib, Genentech and Exelixis, announced subsequently that the combination improved OS, but this was not part of the data submitted to the US Food and Drug Administration for approval. ${ }^{2}$

According to the ASCO analysis, ORR was $69.6 \%$ in the combination arm, compared with $50 \%$ in the vemurafenib and placebo arm, with complete response rates of $15.8 \%$ and $10.5 \%$, respectively, and the median DoR was 12.98 months and 9.23 months. ${ }^{3}$
The safety of cobimetinib in combination with vemurafenib was evaluated in 493 patients who received at least 1 dose. The most common adverse events (AEs) were diarrhea, nausea, vomiting, rash, photosensitivity reaction, hyperkeratosis, fatigue, pyrexia, arthralgia, alopecia, increased alanine transaminase and aspartate transaminase levels, and increased creatine kinase levels. Rates of central serous retinopathy, gastrointestinal events, photosensitivity, increased ALT and AST levels, and increased creatine kinase levels were higher with combination therapy, whereas keratoacanthomas, cutaneous squamous cell carcinoma, alopecia, and arthralgias all occurred at a lower rate. The rates of grade $3 \mathrm{AEs}$ were similar in the 2 groups; however, there were more 
grade 4 events in the combination arm. There was no significant difference in the rate of drug-related discontinuations in the 2 arms.

The recommended dose of cobimetinib, which is marketed as Cotellic by Genentech, is $60 \mathrm{mg}$ orally once daily for the first 21 days of a 28 -day cycle. The prescribing information details recommended dose reductions in the case of adverse events detailed in the warnings and precautions. Patients taking cobimetinib should be monitored for signs and symptoms of new cutaneous and noncutaneous primary malignancies, as well as for hemorrhage, cardiomyopathy, severe dermatologic reactions, serous retinopathy and retinal vein occlusion, hepatotoxicity, rhabdomyolysis, severe photosensitivity, and embryofetal toxicity.

Cobimetinib should be withheld in the case of grade 3 hemorrhagic events and resumed at a lower dose if there is

\section{References}

1. US Food and Drug Administration. FDA approves Cotellic as part of combination treatment for advanced melanoma. US Food and Drug Administration Web site. http://www.fda.gov/NewsEvents/ Newsroom/PressAnnouncements/ucm471934.htm. Accessed December 13, 2015

2. Larkin J, Ascierto PA, Dreno B, et al. Combined vemurafenib and cobimetinib in BRAF-mutated melanoma. N Engl J Med. 2014;371:1867-1876.

3. Larkin JM, Yan Y, McArthur GA, et al. Update of progression-free survival (PFS) and correlative biomarker analysis from coBRIM: Phase III study of cobimetinib plus vemurafenib in advanced BRAF- an improvement to grade 0 or 1 within 4 weeks, or permanently discontinued for those that do not improve or for grade 4 events. Left ventricular ejection fraction (LVEF) should be evaluated prior to and 1 month after starting therapy, and every 3 months thereafter. The safety of cobimetinib has not been established in patients with baseline LVEF below the institutional lower limit of normal or below $50 \%$. Liver lab tests and baseline serum creatine phosphokinase and creatinine levels should be monitored before initiating therapy and periodically during treatment or as clinically indicated. An opthalmological evaluation should be performed regularly or on reports of new or worsening visual disturbances. Patients should be advised to avoid sun exposure, wear protective clothing, and use a broad-spectrum UVA-UVB sunscreen and lip balm, and women of reproductive potential should be warned of the possibility of fetal harm. ${ }^{4}$

mutated melanoma. J Clin Oncol. 33, 2015(suppl; abstr 9006).

4. Cotellic (cobimetinib) tablets, for oral use. Prescribing Information. Genentech Inc. http://www.gene.com/download/pdf/cotellic_prescribing.pdf. Accessed December 13, 2015.

5. Gowrishankar K, Carlino MS, Rizos H. Acquired resistance to targeted MAPK inhibition in melanoma. From Melanoma - early detection to treatment. Ed: Guy Huynh Thien Duc. http://www.intechopen.com/books/melanoma-from-early-detection-to-treatment/ acquired-resistance-to-targeted-mapk-inhibition-in-melanoma. Published January 13, 2013. Accessed December 13, 2015. 